\title{
Reliable RTK Positioning with Tight Coupling of 6 Low-Cost Sensors
}

\author{
Patrick Henkel ${ }^{*, * *}$ and Houcem Hentati * \\ * Technische Universität München (TUM), Munich, Germany \\ ${ }^{* *}$ Advanced Navigation Solutions - ANavS, Munich, Germany
}

\section{BIOGRAPHIES}

Patrick Henkel received his Bachelor, Master and $\mathrm{PhD}$ degrees from the Technische Universität München, Munich, Germany. In 2010, he graduated with a PhD thesis on reliable carrier phase positioning (summa cum laude) and is now working towards his habilitation in the field of precise point positioning. He visited the Mathematical Geodesy and Positioning group at TU Delft in 2007, and the GPS Lab at Stanford University in 2008 and 2010. Dr. Henkel received the Pierre Contensou Gold Medal in 2007, the 1st prize in Bavaria at the European Satellite Navigation Competition in 2010, and the Vodafone Award for his dissertation in 2011. He is one of the founders and currently also the managing director of Advanced Navigation Solutions - ANavS.

Houcem Hentati received the first regional and third national prize for his Baccalauréat in Tunisia. He additionally received the excellence scholarship from the Tunisian government, which enabled his studies in Germany. In 2015, Houcem Hentati received his bachelor degree from the Technische Universität München (TUM) with high distinction. He became also a fellow of the prestigious MaxWeber-Programme in 2015. He is now enrolled in Master studies at the TUM and focuses on microwave engineering and satellite navigation.

\section{ABSTRACT}

A tight coupling of GNSS and inertial measurements is needed for both accurate and reliable positioning. The use of Multi-GNSS is recommended to obtain a sufficient number of visible satellites in any outdoor environment.

In this paper, we perform a joint GPS/ GLONASS ambiguity fixing and a tight coupling of GNSS, 3D accelerometer, 3D gyroscope, 3D magnetometer, barometer and thermometer measurements. As GLONASS uses FDMA, double difference ambiguities are no longer integer-valued.
We derive a transformation for the GLONASS double difference ambiguity term, that recovers the integer property and maintains a full-rank system. The obtained transformation maps the real-valued double difference ambiguity terms into integer-valued double difference ambiguity terms and a common single difference ambiguity term, that is treated as a real-valued parameter.

Low-cost GNSS antennas can not suppress multipath and, therefore, require an estimation of multipath errors. We provide a precise model for multipath, that considers an individual amplitude, code delay, phase shift and Doppler shift for each reflected signal, and include it in our sensor fusion. The magnetometer measurements provide a rough attitude information, which makes them very valuable for robust GNSS attitude ambiguity fixing.

We verified the performance of our sensor fusion in a test drive on a parking lot. The fixed phase residuals were in the order of a few centimeters for both GPS and GLONASS, which indicates a very precise position estimation.

\section{INTRODUCTION}

The highly automatized driving of vehicles requires a both accurate and reliable position and attitude information. A fusion of multiple low-cost sensors is needed. The use of GNSS is attractive as the carrier phase measurements can be tracked with millimeter accuracy and can provide an absolute position information with this high accuracy. PPP/RTK is preferable over RTK as it does not need the exchange of measurements from a reference station. However, precise corrections on atmospheric delays, orbital errors, satellite clock offsets and phase biases with an accumulated error of only a small fraction of the wavelength are needed for fast convergence of the ambiguities and their correct fixing to integer numbers. Additionally, earth tides, satellite phase center offsets and the phase wind-up have to be corrected with a model. An inertial sensor is needed for maintaining an accurate position information during short GNSS signal outages (e.g. below trees and bridges), to cor- 
rect for cycle slips, and to enable a fast ambiguity fixing of newly tracked satellites.

This paper focuses on the position and attitude determination with 3 multi-sensor modules, whereas each module includes a low-cost dual constellation GNSS receiver, a 3D accelerometer, a 3D gyroscope, a 3D magnetometer, a barometer and a thermometer. We perform a tight coupling with an extended Kalman filter.

Fig. 1 shows the principal setup: A multi-sensor module is placed on the front part of the vehicle's roof. A real-time kinematic (RTK) positioning is performed with the measurements of the multi-sensor module and a Virtual Reference Station (VRS). The measurements of the VRS are obtained by interpolation of the measurements of a network of reference stations. A second multi-sensor module is placed on the rear-part of the vehicle to enable attitude determination.
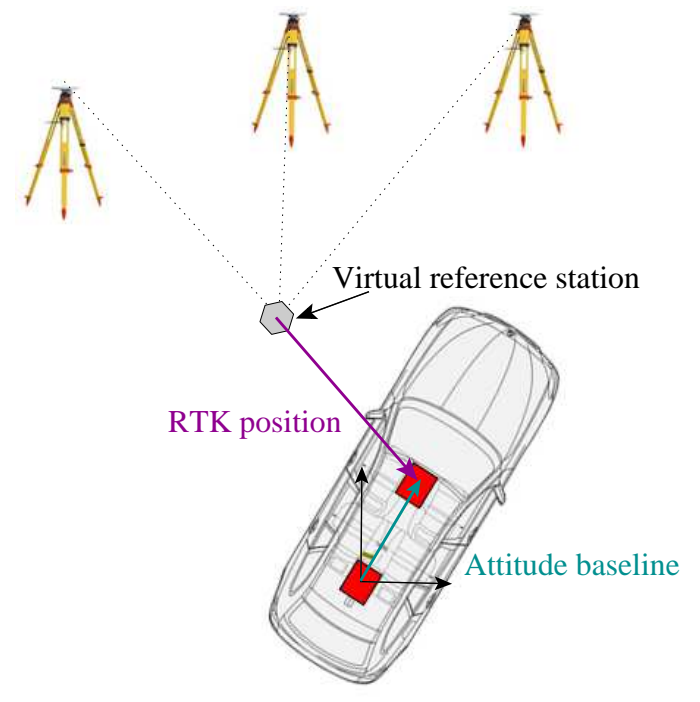

Fig. 1: Measurement setup: Two multi-sensor modules (shown in red) are placed on top of the vehicle for attitude determination. An additional Virtual Reference Station (VRS) is used to estimate the vehicle's position with RTK. The measurements of the VRS are obtained by interpolating the measurements of a network of reference stations.

Fig. 2 shows the principal flow chart for our tightly coupled sensor fusion with integer ambiguity fixing. The position, velocity, acceleration, attitude, angular rates and some further state parameters are predicted using a state space model. The stability of the inertial sensor biases depends on the temperature. Therefore, a thermometer is used to determine the respective process noise statistics.

Subsequently, the state prediction is updated with measurements from the smallest time stamp. These sets of measurements can be either GNSS, inertial (including also magnetic field) or barometric measurements (including air pressure and temperature). Each type of measurement requires some preprocessing: In case of GNSS, this includes the correction for the a priori known satellite positi- ons, clocks and atmospheric delays, the selection of the reference satellite, cycle slip correction, outlier detection and the estimation of noise statistics. For inertial measurements, an estimation of the sensor biases and the frame rotation from the body-fixed frame into the navigation- or ECEF-frame is required. Barometric measurements depend on the weather conditions and need a calibration using a reference station with known air pressure and height.

The (float) ambiguity estimates of the attitude and RTK baselines are fixed to integer numbers, e.g. using the integer decorrelation and tree search of LAMBDA [4]. The search space for the attitude ambiguity fixing can be constrained using baseline length and attitude a priori information (e.g. from a meter and a magnetometer).

Our approach differs in several aspects from the state-ofthe art:

First, we use satellite-satellite single differenced measurements and split the respective ambiguities into a group of absolute (single difference, SD) and relative (double difference, DD) ambiguities. The latter ones are fixed first to obtain a precise attitude information and re-used for the subsequent fixing of the absolute/ SD ambiguities, i.e. both the unfixed SD measurements and the fixed DD measurements of the attitude baselines are considered for the ambiguity fixing of the RTK baseline. The fixing of the attitude baselines is fastened by using soft a priori information on the baseline length and orientation from the magnetometer.

Secondly, we estimate a pseudorange multipath parameter per satellite in both the float and fixed solution to exploit the temporal correlation of multipath and to prevent a mapping of multipath into any other state parameter. The pseudorange multipath error is expressed in terms of the amplitudes and extra-path delays of the reflected signals. We model the time behavior of both the amplitude and extrapath delay of the reflected signal by random-walk processes. This precise modeling of the pseudorange multipath error allows us to reduce the variance of the pseudorange measurements to the variance of the pure noise.

Thirdly, we use the measurements from both GPS and GLONASS to improve the satellite geometry especially in narrow street canyons. The GLONASS ambiguity fixing is more sophisticated than the GPS ambiguity fixing as each satellite is using a different frequency and, thus, the double difference (DD) ambiguities are no longer integer valued. We re-parametrize the $\mathrm{DD}$ ambiguities of $\mathrm{K}$ satellites in terms of K-2 integer valued ambiguities with wavelengths of the respective satellites and one ambiguity combination. The latter one has a wavelength of only a few tens of a $1 \mathrm{~m}$ and, thus, can only be considered as a real-valued parameter. The performance is analyzed in several test drives with 3 ANavS multi-sensor modules and RTK corrections from a Virtual Reference Station (VRS) of Axionet [1].

Fig. 3 shows a multi-sensor module with its 6 low-cost sensors: a GNSS receiver (u-blox LEA M8T) with a patch antenna, a 3D accelerometer, a 3D gyroscope, a 3D ma- 


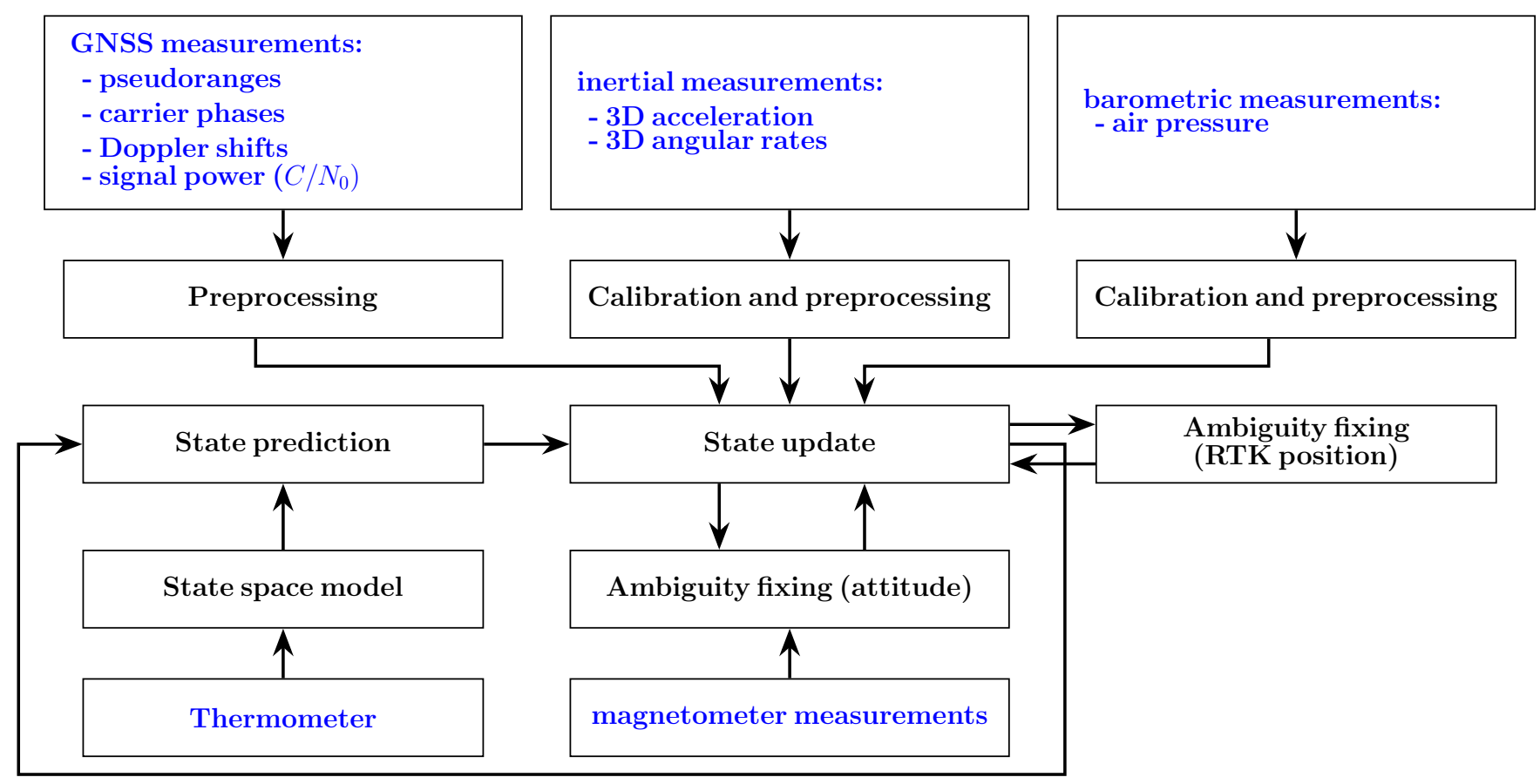

Fig. 2: Flow chart for tightly coupled sensor fusion with six sensors: GNSS, inertial and barometric measurements are preprocessed and used to update the predicted position and attitude. The thermometer improves the state space model. The magnetometer supports the attitude ambiguity fixing.

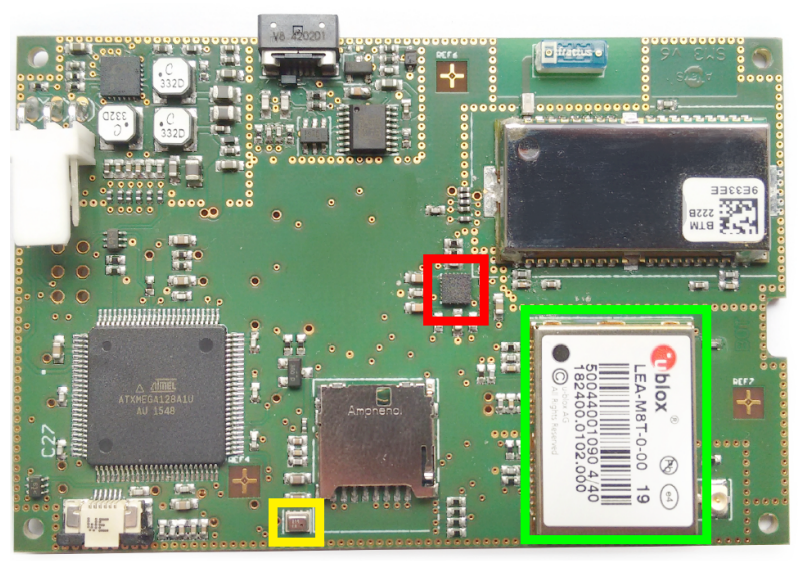

Fig. 3: ANavS Multi-Sensor Module with GNSS receiver (green), 3D accelerometer/ 3D gyroscope and 3D magnetometer (red) and barometer (yellow)

gnetometer (all included in MPU 9250 from Invensense), a barometer and a thermometer (both included in BMP 280 from Bosch Sensortec). We estimate the position, velocity, acceleration, attitude, angular rates, SD ambiguities, code multipath parameters and sensor biases (accelerometer, gyroscope, barometer) as state parameters in our sensor fusion. We obtain a heading accuracy of $0.25 / \mathrm{m}$ and a positioning accuracy of $10 \mathrm{~cm}$ in both urban and rural environments.

\section{MEASUREMENT MODEL}

This section describes our general model for singlefrequency carrier phase measurements from GPS and GLONASS. The undifferenced carrier phase measurement for the $k$-th GNSS satellite of the $r$-th receiver and $i$-th GNSS constellation is modeled according to [2] as

$$
\begin{aligned}
\lambda_{k}^{i} \varphi_{r}^{k, i}= & \left\|\vec{x}_{r}-\vec{x}^{k, i}\right\|+c\left(\delta \tau_{r}^{i}-\delta \tau^{k, i}\right)-I_{r}^{k, i}+T_{r}^{k, i} \\
& +\lambda_{k}^{i} N_{r}^{k, i}+\tilde{\beta}_{r}^{i}\left(n_{k}^{i}\right)+\beta^{k, i}+\varepsilon_{r}^{k, i},
\end{aligned}
$$

with the wavelength $\lambda_{k}^{i}$, the carrier phase measurement $\varphi_{r}^{k, i}$ in units of cycles, the receiver position $\vec{x}_{r}$, the satellite position $\vec{x}^{k, i}$, the speed of light $c$ in vacuum, the receiver clock offset $\delta \tau_{r}^{i}$, the satellite clock offset $\delta \tau^{k, i}$, the ionospheric delay $I_{r}^{k, i}$, the tropospheric delay $T_{r}^{k, i}$, the integer ambiguity $N_{r}^{k, i}$, the receiver phase bias $\tilde{\beta}_{r}^{i}\left(n^{k, i}\right)$ being a linear function of the channel number $n_{k}^{i}$, the satellite phase bias $\beta^{k, i}$ and the phase noise $\varepsilon_{r}^{k, i}$.

For GLONASS measurements, the carrier wavelength is given by

$$
\lambda_{k}^{i}=\frac{c}{f_{0}^{i}+n_{k}^{i} \Delta f},
$$

with $f_{0}^{i}=1602 \mathrm{MHz}$ and $\Delta f=562.5 \mathrm{kHz}$. For GPS measurements, $f_{0}^{i}=1575.42 \mathrm{MHz}$ and $n_{k}^{i}=0 \forall k$.

The receiver phase biases are generated by the receiver's digital signal processing chain (see Sleewaegen et al. [6][7]) and can be accurately modeled as the sum of a 
satellite-independent bias $\beta_{0, r}^{i}$ and a bias $n_{k}^{i} \beta_{r}^{i}$ that is proportional to the channel number (see Wanninger [8]):

$$
\tilde{\beta}_{r}^{i}\left(n_{k}^{i}\right)=\beta_{0, r}^{i}+n_{k}^{i} \beta_{r}^{i}
$$

The single difference (SD) between the measurements of receivers $r$ and $u$ eliminates the satellite clock offset and phase bias, i.e.

$$
\begin{aligned}
\lambda_{k}^{i} \varphi_{u r}^{k, i}= & \vec{e}_{u}^{k, i}\left(\vec{x}_{u}-\vec{x}^{k, i}\right)-\vec{e}_{r}^{k, i}\left(\vec{x}_{r}-\vec{x}^{k, i}\right) \\
& +c \delta \tau_{u r}^{i}-I_{u r}^{k, i}+T_{u r}^{k, i}+\lambda_{k}^{i} N_{u r}^{k, i}+\beta_{u r}^{i}+\varepsilon_{u r}^{k, i} \\
= & \vec{e}_{u}^{k} \vec{x}_{u r}+c_{u r}^{k, i}+c \delta \tau_{u r}^{i}-I_{u r}^{k, i}+T_{u r}^{k, i} \\
& +\lambda_{k}^{i} N_{u r}^{k, i}+\beta_{0, u r}^{i}+n_{k}^{i} \beta_{u r}^{i}+\varepsilon_{u r}^{k, i}
\end{aligned}
$$

with $c_{u r}^{k, i}$ being the synchronization correction between receivers $u$ and $r$ as described in [10] and [9].

The double difference (DD) further eliminates the differential receiver clock offset and phase bias, i.e.

$$
\begin{aligned}
\lambda_{k}^{i} \varphi_{u r}^{k, i}-\lambda_{l}^{i} \varphi_{u r}^{l, i}= & \vec{e}_{u}^{k l, i} \vec{x}_{u r}+c_{u r}^{k l, i}-I_{u r}^{k l, i}+T_{u r}^{k l, i} \\
& +\lambda_{k}^{i} N_{u r}^{k, i}-\lambda_{l}^{i} N_{u r}^{l, i} \\
& +\left(n_{k}^{i}-n_{l}^{i}\right) \beta_{u r}^{i}+\varepsilon_{u r}^{k l, i} .
\end{aligned}
$$

We subtract all available a priori information including the synchronization correction, and the differential ionospheric and tropospheric delay estimates $\hat{I}_{u r}^{k l, i}$ and $\hat{T}_{u r}^{k l, i}$, i.e.

$$
\begin{aligned}
\lambda_{k}^{i} \tilde{\varphi}_{u r}^{k, i}-\lambda_{l}^{i} \tilde{\varphi}_{u r}^{l, i}= & \lambda_{k}^{i} \varphi_{u r}^{k, i}-\lambda_{l}^{i} \varphi_{u r}^{l, i} \\
& -c_{u r}^{k l, i}+\hat{I}_{u r}^{k l, i}-\hat{T}_{u r}^{k l, i} \\
= & \vec{e}_{u}^{k l, i} \vec{x}_{u r}-\Delta I_{u r}^{k l, i}+\Delta T_{u r}^{k l, i} \\
& +\lambda_{k}^{i} N_{u r}^{k, i}-\lambda_{l}^{i} N_{u r}^{l, i} \\
& +\left(n_{k}^{i}-n_{l}^{i}\right) \beta_{u r}^{i}+\varepsilon_{u r}^{k l, i},
\end{aligned}
$$

which leaves the relative position $\vec{x}_{u r}$, the residual atmospheric delays, the integer ambiguities $N_{u r}^{k, i}$ and $N_{u r}^{l, i}$, and the differential receiver phase bias $\beta_{u r}^{i}$ as unknowns. The DD ambiguity term can be rewritten as

$$
\lambda_{k}^{i} N_{u r}^{k, i}-\lambda_{l}^{i} N_{u r}^{l, i}=\lambda_{k}^{i} N_{u r}^{k l, i}+\left(\lambda_{k}^{i}-\lambda_{l}^{i}\right) N_{u r}^{l, i},
$$

with $N_{u r}^{k l, i}$ being the DD integer ambiguity and $N_{u r}^{l, i}$ being the SD integer ambiguity.

The differences of wavelengths can be further developed with $f_{k}^{i}=f_{0}^{i}+n_{k}^{i} \Delta f=\left(n_{0}^{i}+n_{k}^{i}\right) \Delta f$ as

$$
\begin{aligned}
\lambda_{k}^{i}-\lambda_{l}^{i} & =\frac{c}{f_{0}^{i}+n_{k}^{i} \Delta f}-\frac{c}{f_{0}^{i}+n_{l}^{i} \Delta f} \\
& =c \cdot\left(n_{l}^{i}-n_{k}^{i}\right) \frac{\Delta f}{f_{k}^{i} \cdot f_{l}^{i}}=\lambda_{k}^{i} \cdot \frac{n_{l}^{i}-n_{k}^{i}}{n_{0}^{i}+n_{l}^{i}}
\end{aligned}
$$

which is approximately $65 \mu \mathrm{m}$ for $n_{0}^{i}=f_{0}^{i} / \Delta f=2848$ and $n_{l}^{i}-n_{k}^{i}=1$.
Eq. (7) can be written in matrix-vector notation as

$$
\begin{aligned}
& {\left[\begin{array}{c}
\lambda_{1}^{i} N_{u r}^{1, i}-\lambda_{l}^{i} N_{u r}^{l, i} \\
\lambda_{2}^{i} N_{u r}^{2, i}-\lambda_{l}^{i} N_{u r}^{l, i} \\
\vdots \\
\lambda_{k}^{i} N_{u r}^{K, i}-\lambda_{l}^{i} N_{u r}^{l, i}
\end{array}\right]}
\end{aligned}
$$

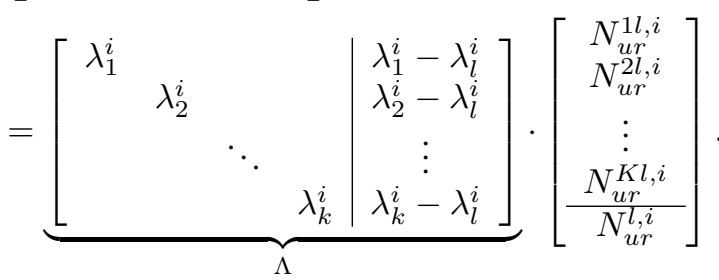

\section{PARAMETER TRANSFORMATION}

The ambiguity coefficient matrix $\Lambda$ has $K+1$ columns but only $K$ rows, which results in a rank-deficient system of equations. Therefore, we map one DD ambiguity (here: the first one $N_{u r}^{1 l, i}$ ) to the SD ambiguity $N_{u r}^{l, i}$ and introduce $\tilde{N}_{u r}^{l, i}$ as

$$
\begin{aligned}
\tilde{N}_{u r}^{l, i} & :=N_{u r}^{l, i}+\frac{\lambda_{1}^{i}}{\lambda_{1}^{i}-\lambda_{l}^{i}} N_{u r}^{1 l, i} \\
& =N_{u r}^{l, i}+\frac{n_{0}^{i}+n_{l}^{i}}{n_{l}^{i}-n_{1}^{i}} N_{u r}^{1 l, i} .
\end{aligned}
$$

The other DD ambiguities have to adjusted respectively, i.e.

$$
\tilde{N}_{u r}^{k l, i}=N_{u r}^{k l, i}-\frac{n_{l}^{i}-n_{k}^{i}}{n_{l}^{i}-n_{1}^{i}} \cdot N_{u r}^{1 l, i} .
$$

Note that both $\tilde{N}_{u r}^{l, i}$ and $\tilde{N}_{u r}^{k l, i}$ are integer valued for any $N_{u r}^{1 l, i}$ only if $n_{l}^{i}-n_{1}^{i}$ is 1 .

Multiplying both the SD and DD ambiguities by $n_{l}^{i}-n_{1}^{i}$ ensures that the ambiguities are always integer valued, i.e.

$$
\begin{aligned}
\bar{N}_{u r}^{l, i} & =\left(n_{l}^{i}-n_{1}^{i}\right) \cdot \tilde{N}_{u r}^{l, i} \\
& =\left(n_{l}^{i}-n_{1}^{i}\right) \cdot N_{u r}^{l, i}+\left(n_{0}^{i}+n_{l}^{i}\right) \cdot N_{u r}^{1 l, i} \in \mathbb{Z} \\
\bar{N}_{u r}^{k l, i} & =\left(n_{l}^{i}-n_{1}^{i}\right) \cdot \tilde{N}_{u r}^{k l, i} \\
& =\left(n_{l}^{i}-n_{1}^{i}\right) \cdot N_{u r}^{k l, i}-\left(n_{l}^{i}-n_{k}^{i}\right) \cdot N_{u r}^{1 l, i} \in \mathbb{Z} .
\end{aligned}
$$

We rewrite Eq. (9) in terms of $\bar{N}_{u r}^{l, i}$ and $\bar{N}_{u r}^{k l, i}$ to obtain the following full-rank ambiguity transformation:

$$
\begin{aligned}
& {\left[\begin{array}{c}
\lambda_{1}^{i} N_{u r}^{1, i}-\lambda_{l}^{i} N_{u r}^{l, i} \\
\lambda_{2}^{i} N_{u r}^{2, i}-\lambda_{l}^{i} N_{u r}^{l, i} \\
\vdots \\
\lambda_{k}^{i} N_{u r}^{K, i}-\lambda_{l}^{i} N_{u r}^{l, i}
\end{array}\right]} \\
& =\left[\begin{array}{ccc|c}
0 & \cdots & 0 & \frac{\lambda_{1}^{i}-\lambda_{l}^{i}}{n_{l}^{i}-n_{1}^{i}} \\
\frac{\lambda_{2}}{n_{l}^{i}-n_{1}^{i}} & & & \frac{\lambda_{2}^{i}-\lambda_{l}^{i}}{n_{l}^{i}-n_{1}^{i}} \\
& \ddots & & \vdots \\
& & \frac{\lambda_{k}}{n_{l}^{i}-n_{1}^{i}} & \frac{\lambda_{k}-\lambda_{l}}{n_{l}^{i}-n_{1}^{i}}
\end{array}\right] \cdot\left[\begin{array}{c}
\bar{N}_{u r}^{2 l, i} \\
\vdots \\
\bar{N}_{u r}^{K l, i} \\
\hline \bar{N}_{u r}^{l, i}
\end{array}\right] .
\end{aligned}
$$


We recommend to choose $n_{l}^{i}$ and $n_{1}^{i}$ such that $n_{l}^{i}-n_{1}^{i}=$ 1. In this case, the combined $K-2$ DD ambiguities $\bar{N}_{u r}^{k l, i}$ can be considered as integer-valued with wavelengths $\lambda_{k}^{i}$, $k \in\{1, \ldots, K\}$, while the SD ambiguity $\bar{N}_{u r}^{l, i}$ is considered as real-valued due to the small differential wavelengths $\lambda_{k}^{i}-\lambda_{l}^{i}$.

The DD carrier phase measurements of Eq. (6) also include the unknown differential receiver phase bias $\beta_{u r}^{i}$, which can not be separated from the ambiguities. Therefore, we map the differential receiver phase bias $\beta_{u r}^{i}$ to the SD ambiguity $\bar{N}_{u r}^{l}$, i.e.

$$
\begin{aligned}
& \frac{\lambda_{k}^{i}-\lambda_{l}^{i}}{n_{l}^{i}-n_{1}^{i}} \cdot \bar{N}_{u r}^{l, i}+\left(n_{k}^{i}-n_{l}^{i}\right) \cdot \beta_{u r}^{i} \\
& =\frac{\lambda_{k}^{i}-\lambda_{l}^{i}}{n_{l}^{i}-n_{1}^{i}} \cdot\left(\bar{N}_{u r}^{l, i}+\frac{\left(n_{k}^{i}-n_{l}^{i}\right)\left(n_{l}^{i}-n_{1}^{i}\right)}{\lambda_{k}^{i}-\lambda_{l}^{i}} \cdot \beta_{u r}^{i}\right) \\
& =\frac{\lambda_{k}^{i}-\lambda_{l}^{i}}{n_{l}^{i}-n_{1}^{i}} \cdot(\bar{N}_{u r}^{l, i}-\underbrace{\frac{\left(n_{0}^{i}+n_{l}^{i}\right)\left(n_{l}^{i}-n_{1}^{i}\right)}{\lambda_{k}^{i}}}_{\gamma_{k}^{i}} \cdot \beta_{u r}^{i}),
\end{aligned}
$$

where the last identity was obtained with Eq. (8). The dependency of the joint SD ambiguity/ bias term on $\lambda_{k}^{i}$ is not desirable as only one SD parameter can be estimated. We replace $\lambda_{k}^{i}$ by $\lambda_{0}^{i}=c / f_{0}^{i}$ in $\gamma_{k}^{i}$ and introduce the error term $\varepsilon^{i}$ :

$\frac{\lambda_{k}^{i}-\lambda_{l}^{i}}{n_{l}^{i}-n_{1}^{i}} \cdot\left(\bar{N}_{u r}^{l, i}-\gamma_{k}^{i} \beta_{u r}^{i}\right) \stackrel{!}{=} \frac{\lambda_{k}^{i}-\lambda_{l}^{i}}{n_{l}^{i}-n_{1}^{i}} \cdot\left(\bar{N}_{u r}^{l, i}-\gamma_{0}^{i} \beta_{u r}^{i}\right)+\varepsilon^{i}$

Solving for $\varepsilon^{i}$ yields

$$
\begin{aligned}
\varepsilon^{i} & =\frac{\lambda_{k}^{i}-\lambda_{l}^{i}}{n_{l}^{i}-n_{1}^{i}} \cdot\left(\gamma_{0}^{i}-\gamma_{k}^{i}\right) \cdot \beta_{u r}^{i} \\
& =\left(\lambda_{k}^{i}-\lambda_{l}^{i}\right) \cdot\left(n_{0}^{i}+n_{l}^{i}\right) \cdot\left(\frac{1}{\lambda_{0}^{i}}-\frac{1}{\lambda_{k}^{i}}\right) \cdot \beta_{u r}^{i} \\
& =\left(\lambda_{k}^{i}-\lambda_{l}^{i}\right) \cdot\left(n_{0}^{i}+n_{l}^{i}\right) \cdot\left(\frac{n_{k}^{i} \Delta f}{c}\right) \cdot \beta_{u r}^{i} .
\end{aligned}
$$

Replacing $\Delta f$ by $f_{l}^{i} /\left(n_{0}^{i}+n_{l}^{i}\right)$ further simplifies $\varepsilon^{i}$ to

$$
\varepsilon^{i}=\frac{\lambda_{k}^{i}-\lambda_{l}^{i}}{\lambda_{l}^{i}} \cdot n_{k}^{i} \cdot \beta_{u r}^{i}
$$

which is far below the millimeter-level for any relevant phase bias $\beta_{u r}^{i}$. Thus, we can neglect $\varepsilon^{i}$ and replace $\gamma_{k}^{i}$ by $\gamma_{0}^{i}$ in Eq. (14).

The DD carrier phase measurements of Eq. (6) can be written with Eq. (13) in matrix-vector notation as

$$
\begin{aligned}
& {\left[\begin{array}{c}
\lambda_{1}^{i} \tilde{\varphi}_{u r}^{1, i}-\lambda_{l}^{i} \tilde{\varphi}_{u r}^{l, i} \\
\lambda_{2}^{i} \tilde{\varphi}_{u r}^{2, i}-\lambda_{l}^{i} \tilde{\varphi}_{u r}^{l, i} \\
\vdots \\
\lambda_{k}^{i} \tilde{\varphi}_{u r}^{K, i}-\lambda_{l}^{i} \tilde{\varphi}_{u r}^{l, i}
\end{array}\right]} \\
& =\left[\begin{array}{cc}
\vec{e}_{u}^{1 l, i} & \frac{\lambda_{1}^{i}-\lambda_{l}^{i}}{n_{l}^{i}-n_{1}^{i}} \\
\vec{e}_{u}^{2 l, i} & \frac{\lambda_{2}^{i}-\lambda_{l}^{i}}{n_{l}^{i}-n_{1}^{i}} \\
\vdots & \\
\vec{e}_{u}^{K l, i} & \frac{\lambda_{k}^{i}-\lambda_{l}^{i}}{n_{l}^{i}-n_{1}^{i}}
\end{array}\right]\left[\begin{array}{c}
\vec{x}_{u r} \\
\bar{N}_{u r}^{l, i}-\gamma_{0}^{i} \beta_{u r}^{i}
\end{array}\right] \\
& +\left[\begin{array}{ccc}
0 & \cdots & 0 \\
\frac{\lambda_{2}^{i}}{n_{l}^{i}-n_{1}^{i}} & & \\
& \ddots & \\
& & \frac{\lambda_{K}^{i}}{n_{l}^{i}-n_{1}^{i}}
\end{array}\right] \cdot\left[\begin{array}{c}
\bar{N}_{u r}^{2 l, i} \\
\vdots \\
\bar{N}_{u r}^{K l, i}
\end{array}\right] \\
& +\left[\begin{array}{c}
\varepsilon_{u r}^{1 l, i} \\
\varepsilon_{u r}^{2 l, i} \\
\vdots \\
\varepsilon_{u r}^{K l, i}
\end{array}\right],
\end{aligned}
$$

where we have assumed that the residual atmospheric errors are negligible.

The DD pseudorange measurements of GLONASS are also affected by inter-frequency biases. We extend the model of [12], and map the DD code bias $b_{u r}^{k l, i}$ to the DD code multipath $\Delta \rho_{\mathrm{MP}_{u r}^{k l, i}}$, i.e.

$$
\begin{gathered}
{\left[\begin{array}{c}
\tilde{\rho}_{u r}^{1 l, i} \\
\vdots \\
\tilde{\rho}_{u r}^{K l, i}
\end{array}\right]=\left[\begin{array}{c}
\tilde{\rho}_{u r}^{1 l, i}-c_{u r}^{1 l, i} \\
\vdots \\
\tilde{\rho}_{u r}^{K l, i}-c_{u r}^{K l, i}
\end{array}\right]} \\
=\left[\begin{array}{ccc}
\vec{e}_{u}^{1 l, i} & 1 & \\
\vdots & \ddots & \\
\vec{e}_{u}^{K l, i} & & 1
\end{array}\right]\left[\begin{array}{c}
\Delta \rho_{\mathrm{MP}_{u r}^{1 l, i}}+b_{u r}^{1 l, i} \\
\vdots \\
\Delta \rho_{\mathrm{MP}_{u r}^{K l, i}}+b_{u r}^{K l, i}
\end{array}\right] \\
+\left[\begin{array}{c}
\eta_{u r}^{1 l, i} \\
\vdots \\
\eta_{u r}^{K l, i}
\end{array}\right]
\end{gathered}
$$

\section{MODELING OF MULTIPATH}

This section briefly describes our multipath model. For a more detailed description, we refer to our earlier paper [3].

We model the received signal at time $t$ as the superposition of the direct signal with amplitude $A_{0}$, code delay $\tau$, phase $\varphi$ and Doppler frequency $\omega$ and $L$ reflected signals with amplitude $A_{l}$, extra path delay $\Delta t_{l}$, phase shift $\Delta \varphi_{l}$ 
and Doppler offset $\Delta \omega_{l}$, i.e.

$$
\begin{aligned}
r(t)= & A_{0} \cdot c(t-\tau) \cdot e^{j(\varphi+\omega(t-\tau))} \\
& +\sum_{l=1}^{L} A_{l} \cdot c\left(t-\left(\tau+\Delta t_{l}\right)\right) \\
& \cdot e^{j\left(\varphi+\Delta \varphi_{l}+\left(\omega+\Delta \omega_{l}\right)\left(t-\left(\tau+\Delta t_{l}\right)\right)\right)}+\eta(t),
\end{aligned}
$$

with $c(t)$ being the chip pulse and $\eta$ being the noise of the received signal. We assume that all tracking loops (DLL, FLL and PLL) are in lock. In this case, we can remove the carrier from the received signal, i.e.

$$
\begin{aligned}
r^{*}(t)= & r(t) \cdot e^{-j(\varphi+\omega(t-\tau))} \\
= & A_{0} \cdot c(t-\tau)+\sum_{l=1}^{L} A_{l} \cdot c\left(t-\left(\tau+\Delta t_{l}\right)\right) \\
& \cdot e^{j\left(\Delta \varphi_{l}-\omega \Delta t_{l}-\Delta \omega_{l} \cdot\left(t-\left(\tau+\Delta t_{l}\right)\right)\right)}+\eta(t) .
\end{aligned}
$$

As the signal of interest is only in the real part of $r^{*}(t)$, we can eliminate the imaginary part of the reflected signals by a projection onto the space of real-valued numbers, i.e.

$$
\begin{aligned}
& \operatorname{Re}\left\{r^{*}(t)\right\}= \\
& A_{0} \cdot c(t-\tau) \\
& +\sum_{l=1}^{L} A_{l} \cdot c\left(t-\left(\tau+\Delta t_{l}\right)\right) \\
& \quad \cdot \cos \left(\Delta \varphi_{l}-\omega \Delta t_{l}-\Delta \omega_{l} \cdot\left(t-\left(\tau+\Delta t_{l}\right)\right)\right) \\
& +\eta(t) .
\end{aligned}
$$

The GNSS receiver correlates $\operatorname{Re}\left\{r^{*}(t)\right\}$ with the receivergenerated signal $A_{0} \cdot c(t-\tau-\Delta)$ and obtains as correlation result:

$$
C(\Delta \tau)=\int_{0}^{T_{\mathrm{c}}} \operatorname{Re}\left\{r^{*}(t)\right\} \cdot A_{0} \cdot c(t-\tau-\Delta \tau) d(t-\tau)
$$

Let us assume that $c(t-\tau)$ is defined as a rectangular pulse, i.e.

$$
c(t-\tau)= \begin{cases}1 & \text { if } 0<t-\tau<T_{\mathrm{c}} \\ 0 & \text { else }\end{cases}
$$

and that the extra-path delays $\Delta t_{l}$ are in the interval $\left[0, T_{\mathrm{c}}\right]$ for all $l$. The correlation result of Eq. (23) can then be further developed as

$$
\begin{gathered}
C(\Delta \tau)=A_{0}^{2} \cdot \int_{\max (0, \Delta \tau)}^{\min \left(\Delta \tau+T_{\mathrm{c}}, T_{\mathrm{c}}\right)} d(t-\tau) \cdot \delta_{\left(\delta \tau<T_{\mathrm{c}}\right)} \\
+\sum_{l=1}^{L} A_{0} A_{l} \cdot I_{l}\left(\min \left(\max \left(\Delta \tau, \Delta t_{l}\right), \Delta t_{l}+T_{\mathrm{c}}\right),\right. \\
\min \left(T_{\mathrm{c}}+\Delta \tau, T_{\mathrm{c}}+\Delta t_{l}\right), \\
\left.\Delta \varphi_{l}, \Delta \omega_{l}, \Delta t_{l}\right)+\tilde{\eta}(t),
\end{gathered}
$$

with $I_{l}$ being defined as

$$
\begin{aligned}
& I_{l}\left(\alpha_{l}, \beta_{l}, \Delta \varphi_{l}, \Delta \omega_{l}, \Delta t_{l}\right) \\
& =\int_{\alpha_{l}}^{\beta_{l}} \cos \left(\Delta \varphi_{l}-\omega \Delta t_{l}-\Delta \omega_{l} \cdot\left(t-\tau-\Delta t_{l}\right)\right) d(t-\tau) \\
& \quad \cdot \delta_{\left(\beta_{l}>\alpha_{l}\right)}+\tilde{\eta}(t),
\end{aligned}
$$

and

$$
\delta_{\left(\beta_{l}>\alpha_{l}\right)}=\left\{\begin{array}{ll}
1 & \text { if } \beta_{l}>\alpha_{l} \\
0 & \text { else. }
\end{array} .\right.
$$

GNSS receivers use a discriminator for tracking the code delay $\Delta \tau$. The Early-minus-Late discriminator [15] is widely used and defined as

$$
\begin{aligned}
D(\Delta \tau) & =C_{\mathrm{E}}(\Delta \tau)-C_{\mathrm{L}}(\Delta \tau) \\
& =C\left(\Delta \tau-d T_{\mathrm{c}} / 2\right)-C\left(\Delta \tau+d T_{\mathrm{c}} / 2\right)
\end{aligned}
$$

with $d T_{\mathrm{c}} / 2, d \leq 1$, being the correlator spacing. We assume without loss of generality that the extra-path delay $\Delta t_{l}$ is smaller than $d T_{\mathrm{c}} / 2$. The discriminator function is continuous in the interval

$$
\Delta \tau \in\left[-\frac{d T_{\mathrm{c}}}{2}+\max _{l}\left(\Delta t_{l}\right), \frac{d T_{\mathrm{c}}}{2}\right] .
$$

We denote the discriminator function at the lower and upper end of this interval by $D_{\mathrm{l}}$ and $D_{\mathrm{u}}$, i.e.

$$
\begin{aligned}
D_{\mathrm{l}} & =C\left(-d T_{\mathrm{c}}+\max _{l}\left(\Delta t_{l}\right)\right)-C\left(\max _{l}\left(\Delta t_{l}\right)\right) \\
D_{\mathrm{u}} & =C(0)-C\left(d T_{\mathrm{c}}\right) .
\end{aligned}
$$

The discriminator function has a zero crossing in this interval. The non-zero code delay at the zero-crossing represents the pseudorange error due to multipath. We approximate the discriminator function by a linear function, i.e.

$$
D(\Delta \tau)=D_{\mathrm{l}}+\frac{D_{\mathrm{u}}-D_{\mathrm{l}}}{\Delta \tau_{\mathrm{u}}-\Delta \tau_{\mathrm{l}}} \cdot\left(\Delta \tau-\Delta \tau_{\mathrm{l}}\right) .
$$

The pseudorange error due to multipath is obtained by setting $D(\Delta \tau)=0$ and by solving it for $\Delta \tau$, i.e.

$$
\Delta \tau_{\mathrm{MP}}=\Delta \tau_{\mathrm{l}}-\frac{D_{\mathrm{l}}}{D_{\mathrm{u}}-D_{\mathrm{l}}} \cdot\left(\Delta \tau_{\mathrm{u}}-\Delta \tau_{\mathrm{l}}\right)
$$

\section{SOFT-CONSTRAINED ATTITUDE AMBIGUITY FIXING}

We use a soft-constrained attitude ambiguity fixing as described in [13] and [14], i.e. soft a priori information on attitude and baseline length is used in the ambiguity fixing.

The attitude Euler angles $\{\varphi, \theta, \psi\}$, the real-valued SD ambiguities $\bar{N}_{u r}^{l, i}-\gamma_{0}^{i} \beta_{u r}^{i}$ and the DD integer ambiguities $\bar{N}_{u r}^{k l, i}, k \in\{2, \ldots, K\}$ are determined jointly by minimizing the sum of squared residual errors (SSE), i.e.

$\min _{\varphi, \theta, \psi, \bar{N}_{u r}^{l, i}-\gamma_{0}^{i} \beta_{u r}^{i}, \bar{N}_{u r}^{2 l, i}, \ldots, \bar{N}_{u r}^{K l, i}} \mathrm{SSE}$, 
with

$$
\begin{aligned}
& \mathrm{SSE}= \\
& \left\|\left(\begin{array}{c}
\lambda \tilde{\varphi}_{u r}^{i} \\
\tilde{\rho}_{u r}^{i}
\end{array}\right)-H\left(\begin{array}{c}
\vec{x}_{u r}(\varphi, \theta, \psi) \\
\bar{N}_{u r}^{l, i}-\gamma_{0}^{i} \beta_{u r}^{i}
\end{array}\right)-A\left(\begin{array}{c}
\bar{N}_{u r}^{2 l, i} \\
\vdots \\
\bar{N}_{u r}^{K l, i}
\end{array}\right)\right\|^{2} \\
& +\frac{(\psi-\bar{\psi})^{2}}{\sigma_{\bar{\psi}}^{2}}+\frac{(\theta-\bar{\theta})^{2}}{\sigma_{\bar{\theta}}^{2}}+\frac{(\varphi-\bar{\varphi})^{2}}{\sigma_{\bar{\varphi}}^{2}}+\frac{\left(\left\|\vec{x}_{u r}\right\|-\bar{l}\right)^{2}}{\sigma_{\bar{l}}^{2}} .
\end{aligned}
$$

The attitude a priori information is obtained from a magnetometer and the baseline length a priori information is derived from a laser. We refer to our earlier paper [11] for the calibration of the magnetometer and the derivation of the a priori information.

\section{TIGHT COUPLING}

An extended Kalman filter [5] is used for joint RTK position and attitude determination with GNSS/ INS/ barometer tight coupling and fixed GPS/ GLONASS DD ambiguities. The state vector includes the following parameters:

- position, velocity and acceleration of the first receiver on the vehicle

- attitude and angular rates of the vehicle

- SD GPS and GLONASS ambiguities of the first receiver on the vehicle

- SD code multipath for each receiver-satellite link

- biases of accelerometer and gyroscope

The SD ambiguities and SD code multipath parameters also include satellite clock/ orbital errors, satellite phase/ code biases and residual atmospheric errors. We denote the state vector as

$$
\begin{aligned}
x=( & \left(\vec{x}_{1 r}\right)^{\mathrm{T}},\left(\dot{\vec{x}}_{1}\right)^{\mathrm{T}},\left(\ddot{\vec{x}}_{1}\right)^{\mathrm{T}}, \varphi, \theta, \psi, \dot{\varphi}, \dot{\theta}, \dot{\psi}, \\
& N_{1}^{l, 1}, \bar{N}_{1}^{l, 2}-\gamma_{0}^{2} \beta_{1}^{2}, \\
& \left(\Delta \rho_{\mathrm{MP}_{1}}^{1}\right)^{\mathrm{T}}, \ldots,\left(\Delta \rho_{\mathrm{MP}_{R}^{k l}}^{1}\right)^{\mathrm{T}},\left(\Delta \rho_{\mathrm{MP}_{r}}^{1}\right)^{\mathrm{T}}, \\
& \left(\Delta \rho_{\mathrm{MP}_{1}}^{2}\right)^{\mathrm{T}}, \ldots,\left(\Delta \rho_{\mathrm{MP}_{R}^{k l}}^{2}\right)^{\mathrm{T}},\left(\Delta \rho_{\mathrm{MP}_{r}}^{2}\right)^{\mathrm{T}}, \\
& \left.\left(b_{\mathrm{a}}^{\mathrm{b}}\right)^{\mathrm{T}},\left(b_{\omega_{\mathrm{ib}}}^{\mathrm{b}}\right)^{\mathrm{T}}\right) .
\end{aligned}
$$

We use the SD carrier phase, pseudorange and Doppler measurements to estimate this state vector and refer to [12] for a detailed description of the relationship between the measurement and state vector. The state vector is predicted with a linear movement model:

$$
\hat{x}_{n}^{-}=\Phi_{n} \hat{x}_{n-1}^{+},
$$

and updated by GNSS, INS and barometric measurements in an alternating manner, i.e.

$$
\hat{x}_{n}^{+}=\hat{x}_{n}^{-}+K_{n}\left(z_{n}^{\mathrm{GPS} / \mathrm{INS} / \mathrm{BARO}}-h_{n}\left(\hat{x}_{n}^{-}\right)\right),
$$

with $h_{n}\left(x_{n}\right)$ describing the non-linear relationship between $x_{n}$ and $z_{n}$. We refer to Jekeli [16] and our earlier paper [12] for a more detailed description of the inertial measurement model.

\section{MEASUREMENT RESULTS}

This section includes a description of our measurement results. We used three ANavS multi-sensor modules and RTK corrections from a Virtual Reference Station (VRS) as described in the introduction. Fig. 4 shows the trajectory of the absolute position as obtained with our multi-sensor RTK solution. The starting point is marked in the left part of the figure.

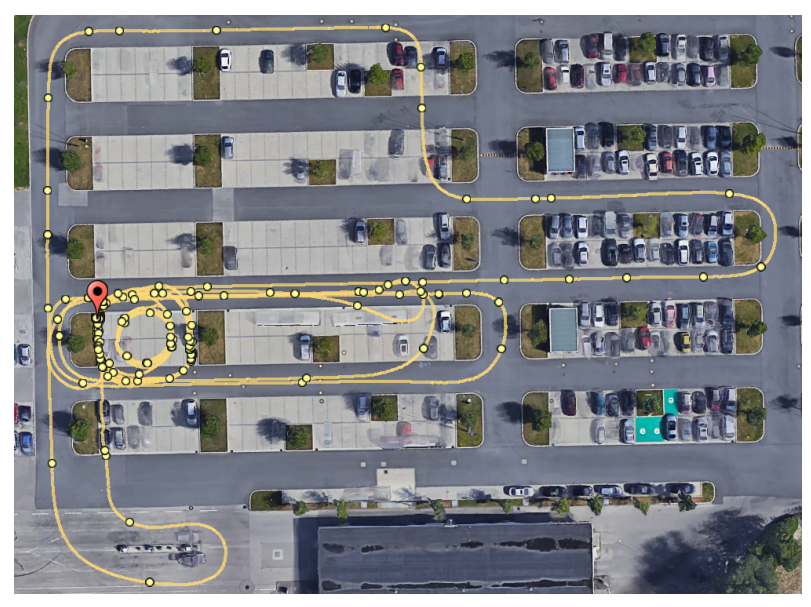

Fig. 4: Map of parking lot showing track of test drive.

Fig. 5 shows the heading of the GPS/ INS tightly coupled solution and the single epoch magnetometer-only heading. One can observe that both heading curves follow each other throughout the complete test drive. However, the heading of the magnetometer is much more noisier than the heading of the GPS/ INS tight coupling.

Fig. 6 shows the difference between the GPS/ INS based heading and the magnetometer heading. The heading offset is less than 25 degrees in all epochs and less than 15 degrees in $95 \%$ of the epochs. Thus, a heading a priori information can be very valuable for attitude ambiguity fixing.

We analyze the benefit of the magnetometer-based heading a priori information for attitude ambiguity fixing based on the time duration for attitude ambiguity fixing.

A fixing is considered as completed once the sum of squared residuals of the best integer candidate is at least three times lower than the sum of squared residuals of the second best candidate. 


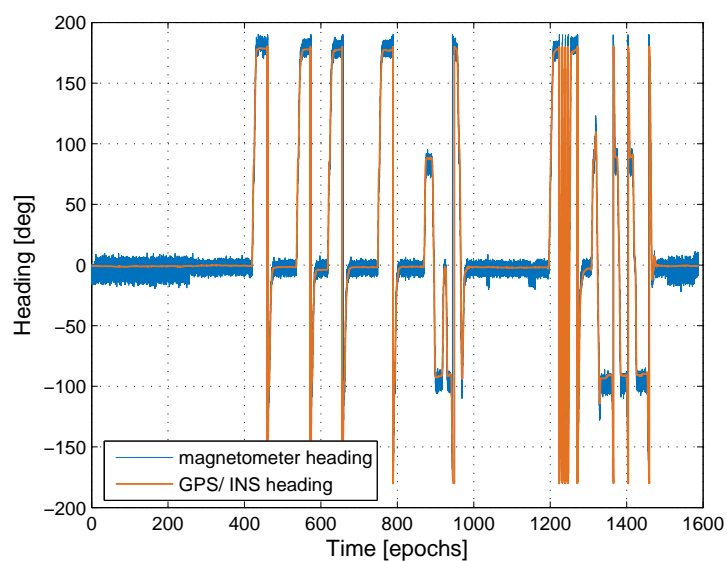

Fig. 5: Comparison of GPS/INS based heading with magnetometer-based heading.

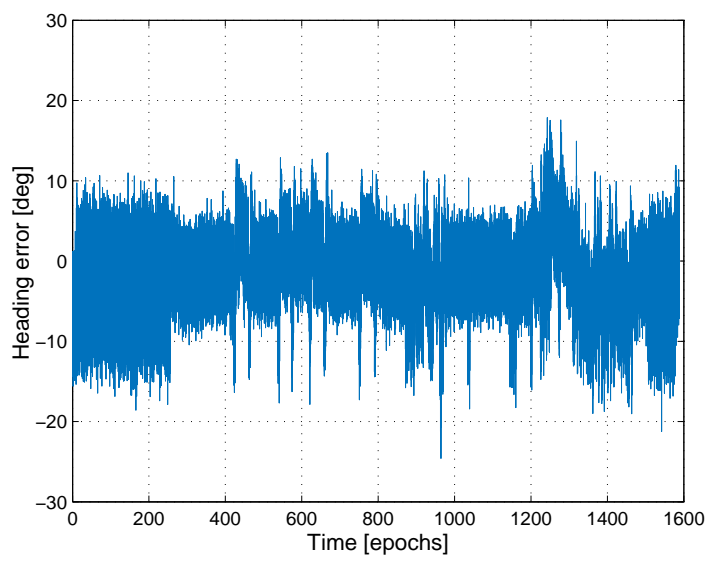

Fig. 6: Difference between GPS/ INS based heading and magnetometer-based heading.

We consider only poor satellite geometries as the magnetometer a priori information is most valuable for these conditions. Poor satellite geometries are obtained by artificially removing two satellites from the set of visible satellites, whereas we consider all possible subsets of two satellites.

Tab. 1 shows the obtained minimum, maximum and mean time for attitude ambiguity fixing with magnetometer-based a priori information. The last column reflects the performance without magnetometer-based heading a priori information and serves as reference. The first two columns show the performance if integer ambiguity candidates with a heading offset of at most $10^{\circ}\left(20^{\circ}\right)$ are used. We can observe that both the maximum and mean time duration can be substantially reduced by the heading a priori information. The threshold of $20^{\circ}$ results in a better average performance than the threshold of $10^{\circ}$ as the error of the heading a priori information is too often above $10^{\circ}$. In this case, the correct integer ambiguity candidate is re- moved from the set of feasible candidates, which results in a poorer performance.

Tab. 1: Performance of attitude ambiguity fixing

\begin{tabular}{l|r|r|r} 
& $|\Delta \bar{\psi}|<10^{\circ}$ & $|\Delta \bar{\psi}|<20^{\circ}$ & $|\Delta \bar{\psi}|<360^{\circ}$ \\
\hline$\Delta t_{\min }$ & $0.2 \mathrm{~s}$ & $0.2 \mathrm{~s}$ & $0.2 \mathrm{~s}$ \\
$\Delta t_{\max }$ & $41.6 \mathrm{~s}$ & $25.6 \mathrm{~s}$ & $481.6 \mathrm{~s}$ \\
$E\{\Delta t\}$ & $5.0 \mathrm{~s}$ & $2.9 \mathrm{~s}$ & $122.0 \mathrm{~s}$ \\
$\sigma(\Delta t)$ & $11.3 \mathrm{~s}$ & $6.1 \mathrm{~s}$ & $141.4 \mathrm{~s}$
\end{tabular}

Fig. 7 and 8 show the fixed phase residuals of both GPS and GLONASS for the RTK baseline. The residuals of all satellites are drift-free with a magnitude of clearly less than 1 cycle, which indicates a correct ambiguity fixing.

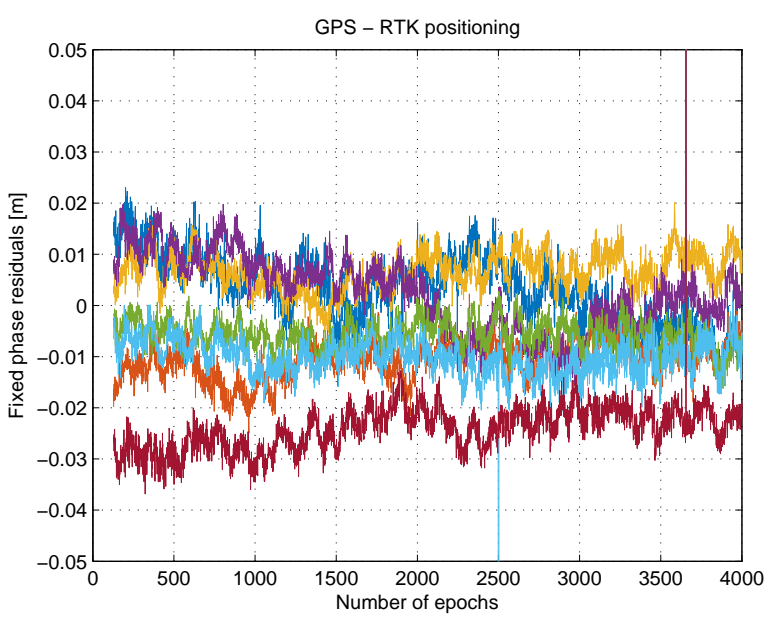

Fig. 7: Residuals of fixed GPS double difference carrier phase measurements for the RTK baseline with a baseline length of $5 \mathrm{~km}$.

The GPS residuals have some small biases while the GLONASS residuals are almost unbiased. This can be explained to some extent by the real-valued scalar parameter $\bar{N}_{u r}^{l}-\gamma_{0} \beta_{u r}$ of GLONASS, which can absorb a common phase bias. Obviously, the residual atmospheric errors are negligible for the considered baseline length of $5 \mathrm{~km}$ as the GLONASS residuals remain unbiased.

\section{ACKNOWLEDGEMENT}

The authors would like to thank the German Federal Ministry of Economic Affairs and Energy (BMWi) and the German Aerospace Center (DLR) for the funding of the project Precise Point Positioning for Autonomous Driving (PPP-AF) with FKZ 50NA1322.

\section{CONCLUSION}

In this paper, we described a tightly coupled sensor fusion of GNSS, 3D acceleration, 3D gyroscope, 3D magne- 


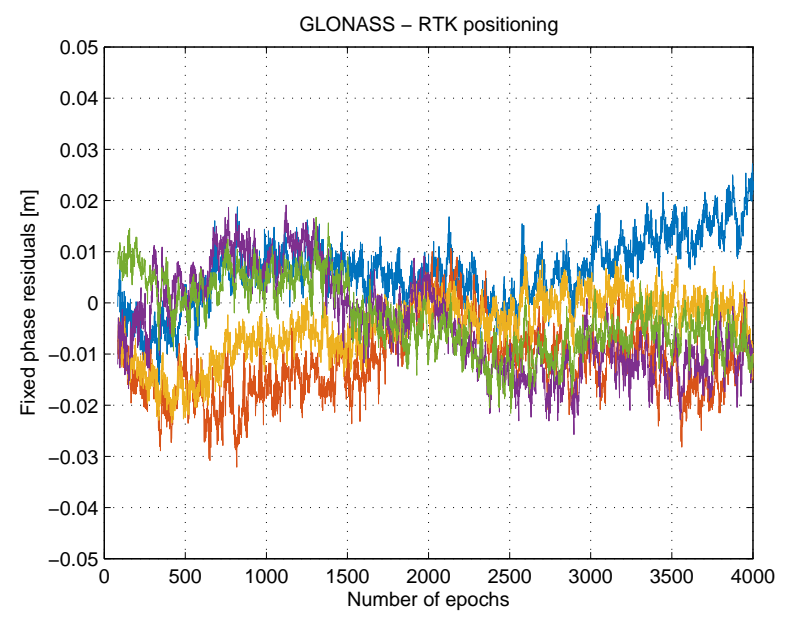

Fig. 8: Residuals of fixed GLONASS double difference carrier phase measurements for the RTK baseline with a baseline length of $5 \mathrm{~km}$.

tometer, barometric air pressure and temperature measurements with integer ambiguity fixing. We derived an ambiguity transformation for FDMA-based GLONASS double difference measurements, that recovers the integer property of ambiguities and that enables a full-rank system. We also provided a precise model for multipath and estimated a multipath parameter per satellite. Measurement results from a test drive showed that the residual errors are in the order of a few centimeters.

\section{REFERENCES}

[1] H. Landau, U. Vollath and X. Chen, Virtual Reference Station Systems, J. of Global Positioning Systems (CGPS), pp. 137 - 143, 2002.

[2] P. Henkel, U. Mittmann and M. Iafrancesco, RealTime Kinematic Positioning with GPS and GLONASS, Proc. of Europ. Signal Processing Conf., Budapest, Sep. 2016.

[3] P. Henkel, M. Iafrancesco and A. Sperl, Precise Point Positioning with Multipath Estimation, Proc. of ION/ IEEE Position, Location and Navigation Symposium, pp. 144 - 149, Apr. 2016.

[4] P. Teunissen, The least-squares ambiguity decorrelation adjustm.: a method for fast GPS integer ambiguity estimation, J. of Geodesy, pp. 65-82, vol. 70, 1995.

[5] R. Brown and P. Hwang, Introduction to Random Signals and Applied Kalman Filtering with Matlab Exercises, 4th edition, Wiley, 2012.

[6] J.-M. Sleewaegen, A. Simsky, W. de Wilde, F. Boon and T. Willems, Origin and Compensation of GLONASS Inter-frequency Carrier Phase Biases in GNSS Receivers, Proc. of 25-th Intern. Techn. Meeting of the Inst. of Navigation (ION), Nashville, USA, pp. 2995 - 3001, Sep. 2012.

[7] J.-M. Sleewaegen, A. Simsky, W. de Wilde, F. Boon and T. Willems, Demystifying GLONASS InterFrequency Carrier Phase Biases, Inside GNSS, pp. 57 - 61, May 2012.

[8] L. Wanninger, Carrier-phase inter-frequency biases of GLONASS receivers, J. of Geodesy, vol. 86, iss. 2, pp. 139 - 148, 2012.

[9] P. Henkel, Tightly coupled Precise Point Positioning and Attitude Determination, IEEE Transactions on Aerospace and Electronic Systems (TAES), vol. 51, no. 4, pp. $3182-3196,2015$.

[10] P. Henkel and M. Iafrancesco, Tightly coupled Position and Attitude Determination with two low-cost GNSS receivers, Proc. of 11-th Intern. IEEE Symp. on Wireless Communication Systems (ISWCS), Barcelona, Spain, pp. 895 - 900, Aug. 2014.

[11] P. Henkel, P. Berthold and J. Kiam, Calibration of Magnetic Field Sensors with two mass-market GNSS receivers, IEEE Proc. of 11-th Workshop on Positioning, Navigation and Communication (WPNC), pp. 1 - 5, Dresden, Germany, Mar. 2014.

[12] P. Henkel and A. Sperl, Precise RTK Positioning with GPS/ INS Tight Coupling and Multipath Estimation, Proc. of the 2016 Intern. Techn. Meeting of the ION, Monterey, USA, pp. 1015 - 1023, 2016.

[13] P. Henkel, P. Jurkowski and C. Günther, Differential Integer Ambiguity Resolution with Gaussian a priori knowledge and Kalman filtering, Proc. of 24th ION GNSS, Portland, USA, pp. 3881 - 3888, Sep. 2011.

[14] P. Henkel and C. Günther, Reliable Integer Ambiguity Resolution: Multi-Frequency Code Carrier Linear Combinations and Statistical A Priori Knowledge of Attitude, Navigation, vol. 59, nr. 1, pp. 61 - 75, 2012.

[15] B. Parkison, J. Spilker, P. Axelrad and P. Enge, Global Positioning System: Theory and Applications, vol. I and II, AIAA, 1996.

[16] C. Jekeli, Inertial Navigation Systems with Geodetic Applications, 352 pp., Walter de Gruyter, 2001. 